\title{
Designing soil tillage devices for the spiral land cultivation system
}

\author{
Sergey Strekalov ${ }^{1, *}$ and Lubov Strekalova ${ }^{2}$ \\ ${ }^{1}$ Volgograd State Technical University, Volgograd, Russia \\ ${ }^{2}$ Volgograd State Agrarian University, Volgograd, Russia
}

\begin{abstract}
The paper considers ways of implementing scientific and technological achievements in crop farming by focusing on soil tillage and plant management operations. It studies requirements to soil tillage device designs and lists efficiency criteria for their operation. Theoretical research is being conducted into finding monoprinciples for creating multifunctional devices for the spiral crop farming system. A synthesis of principally new wave-motion-based tools is presented. The critical operational characteristics of wave-type tools and their technological aspects are given.
\end{abstract}

The current state of agricultural production in Russia is characterized by decreased efficiency as a result of a number of objective and subjective reasons, which, first and foremost, include the low technical and technological support level of agriculture. This, in turn, has led to a drop in profitability of agriculture, and consequently, to a collapse and bankruptcy of agricultural companies, as well as an outflow of rural population, which has led to lower staffing levels in all industries against the backdrop of decreasing birth rates and population figures in rural areas. It is noteworthy that the number of workers in the agro-industrial sector of Russia is 7\% (as compared to 2\% in the USA, and 5\% in the EU). Only $1 \%$ of agricultural companies and farms existing in Russia operate with a high level of innovative technology, and the innovative potential of the Russian agro-industrial sector is generally utilized by $4 \%$, whereas in the USA the figure is $50 \%$ [1].

The need for innovation in agriculture by using knowledge-intensive technologies is dictated by the modern demands of agricultural production. Implementing technological advances in agriculture is a long-term trend. According to the Food and Agriculture Organization of the United Nations (FAO), provision of food to global population in the nearest century will be accomplished through innovative resources in agriculture and intelligent improvement of agricultural technologies [2].

In the current situation of low productivity in Russia's agro-industrial sector, there is an acute need to develop land cultivation systems which will ensure a gradual saturation of the internal food market. By implementing the State Program outlining the areas for improvements in the science and technology policy, it will be possible to upgrade, develop and maintain the innovative course of development in the agro-industrial sector in 20132020 [3].

\footnotetext{
* Corresponding author: strekalov sergey@mail.ru
} 
In cultivating any agricultural plant, one can identify the following basic operations: soil tillage, sowing, crop tending, harvesting and commercial enhancement of the yield.

Soil tillage uses different principles and methods of processing a soil layer or its part by applying tools and can be divided into a number of groups depending on the method applied. For example, these methods may include tillage with soil overturning or without it, thorough mixing (special techniques), deep tillage, etc. Further, each processing method uses its own techniques and tools to achieve certain soil characteristics, upper layer uniformity, a cetain hydrophysical mode of the processed layer, etc. [4, 5].

The previously most common system, i.e. the moldboard plow system, does not ensure surface uniformity, creates a plow pan, and is susceptible to wind and water erosion due to the absence of non-productive organic matter on the surface.

Flat-cutting tillage does not facilitate accumulation and storage of moisture because the dense plow pan created by blade plows inhibits its penetration into the substrate.

The no-till system where the soil is not tilled does not ensure moisture preservation and leads to an increased level of soil consistency density and a gradual accumulation of weed vegetation. It is also worth noting that the decreased intensity of soil tillage in the no-till system may potentially reduce its environmental impact [6].

The strip-till technology is a tillage method where cutting guide slots in the soil for a repeated run of seeding mechanisms occurs, combining the advantages of zero tillage and traditional plowing. [7]. This technology considers different ways of tool orientation during soil tillage and crop tending. One of these methods is orientation as per the precut slot, which allows decreasing the protection zone size and improving the movement accuracy during soil tillage operations. In this method, rotational soil tillage machines are preferred $[8]$.

One drawback of this tilling mechanism orientation method is the significant consumption of energy required for cutting the guide slot.

When designing soil tillage equipment for any land cultivation technology, the main objective is to ensure that the tool leaves a stubble on the field surface, does not create a plow pan and meets the agrotechnical requirements with respect to lumpiness, uniformity and moisture retention in the soil.

The general criteria of effective operation of technical agricultural facilities are the minimal movement time, minimal energy consumption for movement and minimal force to perform an operation.

The wide range of tools and the requirements imposed on them make the design of the universal soil tillage system considerably more complex. For this reason, it is necessary to look for monoprinciples which would make it possible to simplify the design and increase its reliability when creating multifunctional tools.

Thus, researching and developing this area should lead to savings in energy, time and materials, which is one of the oldest principles of theoretical science resulting in economy and optimality.

The current state of tilling machines is lagging behind these requirements, which have to ensure that the soil structure is preserved to the greatest possible extent, erosion is diminished, and the potential of the cultivated crop is utilized to the full. Active drive machines are consistent with these requirements to a larger degree.

It is necessary to create principally new tools based on improved motion modes. Wave motion is the main type used in nature to propel through different media. In this respect, the application of multilink wave surfaces in synthesizing wave-type soil tillage tools is of considerable interest.

Implementing the optimality principles in devising universal mechanisms interacting with a solid medium is characterized by one essential feature - the individuality of the multilink wave surface is used in developing the soil tilling machine design [9]. 
The wave-type technical devices which have been created are adapted to the tillage process and plant handling to the greatest possible degree and allow soil tillage and weeding operations in the spiral crop farming while ensuring the simultaneous orientation of the robotic system during its movement in the field [11].

Let us consider the interaction between the wave surface link and the continuous medium, when the link performs forced vibrations which have a certain impact on the continuous medium. The following equation can describe the motion in the continuous medium in a vector form (Navier-Stokes equation):

$$
\frac{d v}{d t}=F-\frac{1}{\rho} \operatorname{grad} p+\frac{\lambda_{c}+\mu}{\rho} \operatorname{graddi} v v+v \Delta v,
$$

where $\mathrm{F}$ is the mass force field strength; $p$ is the pressure; $\rho$ is the medium density; $v$ is the kinematic medium viscosity; $\Delta v$ is the Laplace operator:

$$
\Delta v=\frac{\partial^{2} t}{\partial x^{2}}+\frac{\partial^{2} t}{\partial y^{2}}+\frac{\partial^{2} t}{\partial z^{2}}
$$

If the soil tillage machine velocity is $v_{a}$ and the deformation propagation rate is $\mathrm{V}$, given that $\mathrm{V}>>v_{\mathrm{a}}$, there will be an area in front of the link, in which the medium velocity will vary from $v_{\max }$ to $v_{\min }$. According to the above equation, pressure will also change in that area, where it will have a maximum value in the middle of the link and will reduce nearer to the edges. Therefore, a kind of field will be created in front of the link, with its pressure gradient directed perpendicular to the direction of motion.

According to Lyapunov, the equilibrium $X=0$ is called stable if for any $\quad \varepsilon>0$ it is possible to find $\delta>0$, which will result in inequation ||$X(t)||<\varepsilon \mid$, given $\mid X($ to $)||<\delta$ at any $\mathrm{t}>$ to. Otherwise, the equilibrium $\mathrm{X}=0$ is called unstable.

The analysis of the above system shows that according to Lyapunov it is unstable and can deviate from the equilibrium position even if the initial perturbations are negligibly small.

It follows that if the MWS link in the equilibrium state was located in parallel with the direction of the machine motion, it will move away from that position regardless of how small the perturbation is. It should be noted that the deviation from the equilibrium state will be accompanied by a change in the link tilt angle, which is determined according to the following formula for an n-link at small angles:

$$
\alpha \mathrm{i}=\frac{\grave{A}}{L}\{\sin [\omega \mathrm{t}+(\mathrm{i}-1) \varphi]-\sin (\omega \mathrm{t}+\mathrm{i} \varphi)\} .
$$

Therefore, the link can perform oscillations from the highest position to the lowest position under the action of a continuous draft force applied by the machine, i.e. autooscillations will occur, whose frequency is determined by the machine velocity and wave surface parameters.

The drag force may be determined by the following formula:

$$
\mathrm{R}=\mathrm{C}_{\mathrm{x}} \mathrm{S} \frac{\rho v^{2}}{2},
$$

where $\mathrm{C}_{\mathrm{x}}$ - is a non-dimensional coefficient and $\mathrm{S}$ is the link area. 
Under the draft force action, the MWS will perform a wave motion, which will result in the knife of the soil-tilling machine moving from the highest to the lowest position, i.e. the MWS will function due to the use of the applied force (Fig.1).

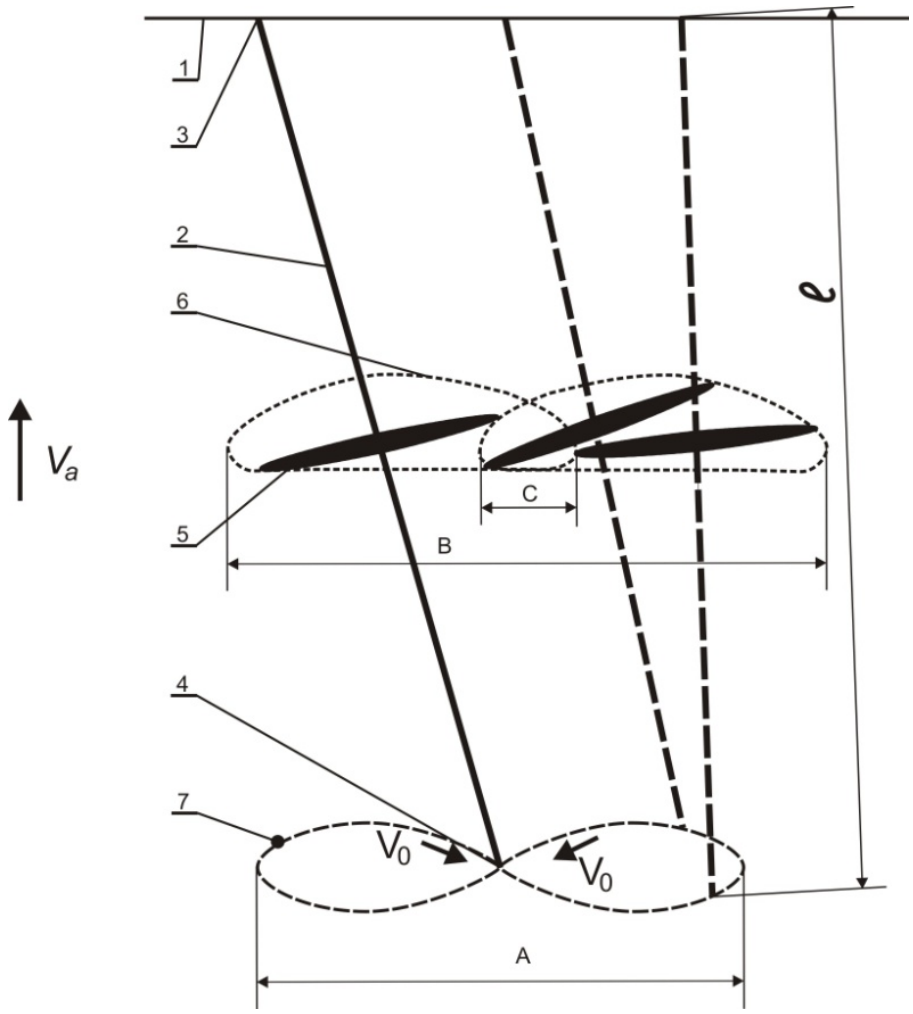

Fig. 1. Interaction between the knife and soil:

1 - basic oscillator motion trajectory; 2 - activator (MSW link); 3 - basic oscillator; 4 - pulse oscillator; 5 - knife; 6 - knife motion trajectory in the soil; 7 - pulse oscillator motion trajectory

As applied to soil tillage, we use the following terminology and designations:

- the wave surface link, - activator 2 (Fig.1);

- the tool acting directly on the soil, - knife 1 (the knife has two working sides, i.e. two cutting edges and two trajectories 7 - right and left);

- the oscillator performing oscillations perpendicular to the movement direction, - basic;

- the oscillator performing oscillations along the machine motion $\mathrm{d}$, - pulse.

In designing soil-tilling tools based on the property of individuality of wave surfaces, we should consider a few options with distinctive design features as to the character of the MSW node motion and the pulse type:

- node motion in the horizontal plane (the wave motion coincides with the machine motion direction, using the macropulsation effect, Fig. 2a, when the amplitude of oscillations is commensurate with the activator length);

- node motion in the vertical plane (the wave direction coincides with the machine motion direction, using macropulsation, Fig. 2b);

- node motion in the horizontal plane (the wave direction coincides with the machine motion direction, using macropulsation, Fig. 2c, when the amplitude is considerably smaller than the activator length). 


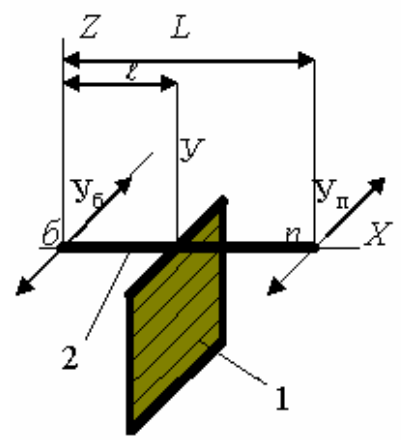

$\mathbf{a}$

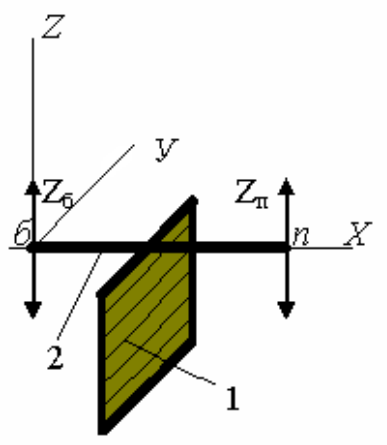

б

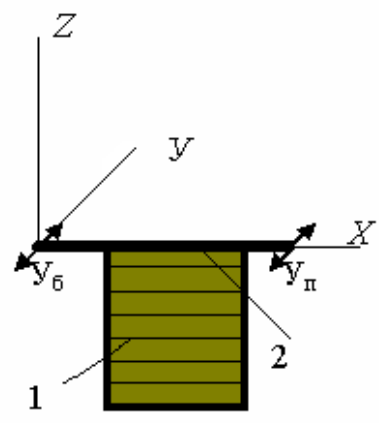

B

Fig. 2. Wave soil-tilling tool layout:

1 - knife; 2 - activator; $\mathrm{Y}_{\bar{\sigma}}, \mathrm{Z}_{\sigma}$ - oscillation magnitude of the basic oscillator; $\mathrm{Y}_{п}, \mathrm{Z}_{п}-$ oscillation magnitude of the pulse oscillator

Due to the summation of phase-displaced oscillations, the knife performs a complex motion consisting of relative (rotation around the vertical axis) and oscillatory motions in the horizontal plane, as well as the linear motion of the machine. The knife motion in the soil is determined by the complex wave motion resulting from the relative and translational motions.

The soil motion trajectory of the tool whose node motion occurs in the horizontal plane (the wave direction coincides with the machine motion direction) is shown in Fig. $2 a$. The knife is attached to the activator with a length of $\mathrm{L}$ and at a distance of $\ell$ from the basic oscillator, which performs harmonic oscillations with amplitude A and angle velocity $\omega$.

The basic oscillator «б» of the activator performs oscillations described by the following set of equations:

$$
\begin{aligned}
& \mathrm{X}_{\sigma}=0, \\
& \mathrm{y}_{\sigma}=\mathrm{A} \sin \omega \mathrm{t} .
\end{aligned}
$$

The pulse oscillator «n» of the activator will follow a trajectory described by the following parametric form equation:

$$
\begin{gathered}
\mathrm{X}_{\Pi}=\mathrm{L} \cos \alpha, \\
\mathrm{y}_{\Pi}=A \sin (\omega \mathrm{t}+\varphi)+\mathrm{L} \sin \alpha,
\end{gathered}
$$

where $\mathrm{L}$ is the activator length, $\mathrm{A}$ is the amplitude of oscillations, $\omega$ is the angle velocity and $\alpha$ is the activator tilt angle.

The motion direction of the tool cutting edges at different $h$ values varies considerably. When $\mathrm{h}>\mathrm{A}$, the trajectories overlap, i.e. in this case the grip zone which will be tilled is determined by the following formula:

$$
\amalg=2(\mathrm{~A}+\mathrm{h}) .
$$




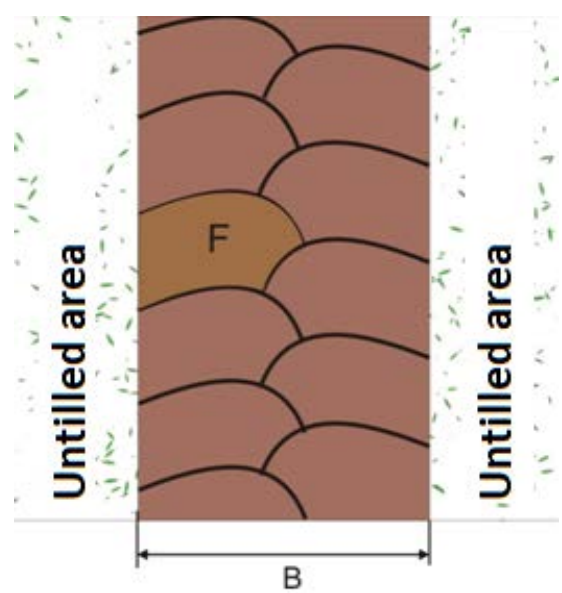

Fig. 3. Knife motion trajectory in the soil:

$\mathrm{B}$ - grip width; $\mathrm{F}$ - step width

The motion trajectory of the tool knife is oval in shape and has a clear rectangular section, with the convexity directed towards the basic oscillator (Fig. 3). This form of trajectory is characteristic of the central activator point.

The velocity will change as the width and position of the knife on the activator change. The highest cutting speed corresponds to the greatest trajectory length.

In the layouts of the soil tillage machines whose oscillators perform oscillations in the vertical plane or in the horizontal planes and whose wave direction coincides with the machine motion direction, the activator and tool motion trajectories are similar, with the only difference being whether only one tool blade is involved in the operation or both sides take part in it.

In the layout of the soil tillage machine where the nodes perform motion in the horizontal plane and the wave direction coincides with the machine direction, provided that the oscillation amplitude value is considerably lower than the activator length, its motion character in the soil is consistent with the wave para-plow operation. Narrow slots are made in the soil, forming better conditions for the supply of water to the root system of the plants and acting as guides for repeated machine runs in the spiral crop cultivation system, in order to ensure a minimal protective zone for plants while weeding cultivated crops.

The cutting speed is influenced by the design features of the machine, such as the knife width and its position on the activator (the knife width is not to exceed the oscillation amplitude) and the wave propagation speed, which increases as the oscillation frequency rises and the pulsation falls, and decreases as the phase shift increases.

This paper has been prepared under the sponsorship of RFBR and the Administration of Volgograd Region; Project No. 19-48-340004

\section{References}

1. L.N. Kosyakova. SPbGAU News Bull, 174-180, (2015)

2. Russian Federation Government Resolution No. 717 of 14 July, 2012 "On the state program of agricultural development and the market of agricultural products, raw materials and food for 2013-2020" (as amended). URL: http://www.consultant.ru/

3. Edict of the President of Russia No. 120 of 30 January, 2010, "On the approval of the Food Safety Doctrine of the Russian Federation”. URL: http://www.consultant.ru/; 
4. V.I. Solodun. Adv. in agroind. scien. and tech. 12, 6-8 (2011)

5. E.P. Yatsuk, et al. Rotational soil-tilling machines. Construction, calculation and design (Mashinistroyenie, Moscow, 1971)

6. R.Q. Cannell. Soil and tillage. 5, 129-177 (1985)

7. T.J. Townsend, S.J. Ramsden, P. Wilson. Soil Manag. 32 (1), 106-117 (2016) (published online)

8. O.V. Vernyayev. Active cultivator tools (Mashinostroyenie, Moscow, 1983)

9. S.D. Strekalov, G.M. Misharev, L.P. Strekalova. Wave technology (Peremena, Volgorad. 2004)

10. S. Strekalov, L. Strekalova, M. Pertsev. MATEC Web of Conf. 224 (2018). International Conference on Modern Trends in Manufacturing Technologies and Equipment (ICMTME 2018) (Sevastopol, Russia, 10-14 September). Article Number 05005

11. S.D. Strekalov, Yu.G. Gordienko, S.E. Grekov, V.A. Polyakov. Farm Mach. Operator J. 9, $18-20$ (2009) 